\title{
Ictal networks of temporal lobe epilepsy: views from high-frequency oscillations in stereoelectroencephalography
}

\author{
Syu-Jyun Peng, PhD, ${ }^{1,2}$ Chien-Chen Chou, MD, ${ }^{3,5}$ Hsiang-Yu Yu, MD, ${ }^{3,5}$ Chien Chen, MD, ${ }^{3,5}$ \\ Der-Jen Yen, MD, ${ }^{3,5}$ Shang-Yeong Kwan, MD, ${ }^{3,5}$ Sanford P. C. Hsu, MD, ${ }^{4,5}$ Chun-Fu Lin, MD, 4,5 \\ Hsin-Hung Chen, MD, ${ }^{4,5}$ and Cheng-Chia Lee, MD, PhD ${ }^{4,5}$
}

\begin{abstract}
1Biomedical Electronics Translational Research Center and ${ }^{2}$ Institute of Electronics, National Chiao-Tung University, Hsinchu; Departments of ${ }^{3}$ Neurology and ${ }^{4}$ Neurosurgery, Neurological Institute, Taipei Veterans General Hospital; and ${ }^{5}$ School of Medicine, National Yang-Ming University, Taipei, Taiwan
\end{abstract}

\begin{abstract}
OBJECTIVE In this study, the authors investigated high-frequency oscillation (HFO) networks during seizures in order to determine how HFOs spread from the focal cerebral cortex and become synchronized across various areas of the brain.
\end{abstract}

METHODS All data were obtained from stereoelectroencephalography (SEEG) signals in patients with drug-resistant temporal lobe epilepsy (TLE). The authors calculated intercontact cross-coefficients between all pairs of contacts to construct HFO networks in 20 seizures that occurred in 5 patients. They then calculated HFO network topology metrics (i.e., network density and component size) after normalizing seizure duration data by dividing each seizure into 10 intervals of equal length (labeled I1-I10).

RESULTS From the perspective of the dynamic topologies of cortical and subcortical HFO networks, the authors observed a significant increase in network density during intervals I5-|10. A significant increase was also observed in overall energy during intervals $13-18$. The results of subnetwork analysis revealed that the number of components continuously decreased following the onset of seizures, and those results were statistically significant during intervals I3-I10. Furthermore, the majority of nodes were connected to a single dominant component during the propagation of seizures, and the percentage of nodes within the largest component grew significantly until seizure termination.

CONCLUSIONS The consistent topological changes that the authors observed suggest that TLE is affected by common epileptogenic patterns. Indeed, the findings help to elucidate the epileptogenic network that characterizes TLE, which may be of interest to researchers and physicians working to improve treatment modalities for epilepsy, including resection, cortical stimulation, and neuromodulation treatments that are responsive to network topologies.

https://thejns.org/doi/abs/10.3171/2018.6.JNS172844

KEYWORDS brain connectivity; graph theory; epileptogenic network; topology; epilepsy surgery

$\mathrm{T}$ He standard approach to improve surgical outcomes in patients with drug-resistant epilepsy involves noninvasive evaluation prior to surgery in order to locate the epileptogenic zone (EZ). However, in some cases, the EZ is not readily identified, such that invasive electroencephalography (iEEG) monitoring is required. Such iEEG procedures include electrocorticography via subdural grid and stereoelectroencephalography (SEEG) via stereotactic depth electrodes. Since 1974, when SEEG was introduced in Paris, ${ }^{6,29}$ epileptologists have actively sought to identify the specific brain regions involved during seizures; however, this has typically relied on visual inspection. With the progress of computer technology and mathematical analysis, epileptologists now can use more computer-assisted quantitative analysis on iEEG data to reduce bias due to limitations in the experience and to facilitate the identification of the EZ. ${ }^{1}$ In addition, the computer-assisted analysis may be able to help explore the ictal networks and even epileptogenic networks.

The use of SEEG to identify the EZ in patients with

ABBREVIATIONS EEG = electroencephalography; EZ = epileptogenic zone; iEEG = invasive EEG; HFO = high-frequency oscillation; SEEG = stereoelectroencephalography; $T L E=$ temporal lobe epilepsy.

SUBMITTED November 21, 2017. ACCEPTED June 27, 2018.

INCLUDE WHEN CITING Published online November 30, 2018; DOI: 10.3171/2018.6.JNS172844. 
drug-resistant epilepsy has become increasingly popular in the last decade. The exploration of dynamic topologies makes it possible for SEEG to delineate the hypothetical epileptic network in 3 dimensions using multiple intracerebral electrodes. Therefore, cortical structures that have been implicated in the generation and early propagation of seizures can be mapped with precise stereotactic guidance, and dynamic topologies of cortical and subcortical functional networks can be established.

Since 2004, there has been more and more evidence showing that high-frequency oscillations (HFOs; 80-500 $\mathrm{Hz}$ ) are related to epileptogenesis..$^{14,15,33}$ As biomarkers for the EZ, HFOs are superior to epileptiform discharges, such as spikes or sharp waves. ${ }^{13,34}$ Furthermore, resecting the regions with HFOs has been shown to yield better seizure outcomes than resecting of the seizure-onset zone alone.$^{13}$ However, there is still a lack of information about the onset, propagation, and termination of HFOs in temporal lobe epilepsy (TLE). To explore the HFO networks during seizures in TLE, we investigated HFOs shown in SEEG data obtained from patients with drug-resistant TLE. In this study, SEEG with a high sampling rate provides us sufficient time resolution to examine HFOs. In addition, SEEG provided dynamic changes that occur over the course of a seizure between various brain areas. By identifying ictal HFO networks that are common in TLE, our hope is to develop a new approach for surgery or neuromodulation to improve epilepsy outcomes.

\section{Methods \\ Patients}

Five patients with TLE (mean age 24 years; range 1648 years) with a median seizure history of 11 years (range 2-17 years) were retrospectively analyzed. All 5 patients were diagnosed as drug-resistant epilepsy (DRE) while taking at least 2 full-dose anticonvulsants. We collected data from the presurgical evaluations, diagnostic studies, surgical strategy, and postoperative follow-up concerning functional morbidity and seizures (median follow-up 28 months; range 24-46 months).

Only 1 patient showed potential epileptogenic lesions on preoperative MRI, and others were MRI negative in a phase I study. For the patient with definite MRI-documented lesions, iEEG (phase II study) was arranged due to discordance of semiology and scalp EEG findings. For patients with MRI-negative findings, the phase II study was recommended for better localization and lateralization. Since 2013, we have performed SEEG in place of iEEG (phase II study).

This study was approved by the Taipei Veterans General Hospital Review Board.

\section{Procedure for SEEG Electrode Implantation}

According to the results of the semiology, image evaluation, scalp EEG, and other presurgical evaluation, individualized SEEG implantation was designed. For each SEEG design, the neurosurgeon usually has 1-3 hypotheses, and the location of SEEG leads are responsible for proving the hypothesis. The SEEG leads have to cover the potential EZ, the border of the resection, and functional area for mapping.
On the day of SEEG electrode implantation, all patients underwent stereotactic frame placement in the operating room while receiving monitored intravenous anesthesia and then underwent stereotactic treatment planning with MRI. The target, entry point, and trajectory were checked using the stereotactic planning workstation (SurgiPlan, Elekta). The patient was then sent to operating room, where stereotaxy-guided depth electrode placement took 2 hours to complete. Intraoperatively, the electrode position can be confirmed using the $\mathrm{O}$-arm, to avoid the malpositioning of electrode bending. When surgery of electrode placement is finished, the patient was sent to a 24-hour monitoring room for video-SEEG recording. For the 5 patients in the study, we placed a total of 42 electrodes (mean 8.4 electrodes). The mean electrode length in the brain was $42.8 \mathrm{~mm}$. The entry point localization error was $1.0 \mathrm{~mm}$ on average, and the target point localization error was $3.6 \mathrm{~mm}$ on average. The mean thickness of scalp skin was $14 \mathrm{~mm}$, and that of scalp bone was $12 \mathrm{~mm}$. The angle for insertion of each electrode was $85^{\circ}$, and the bolts that were used were $13 \mathrm{~mm}$ and $21 \mathrm{~mm}$.

\section{Acquisition of SEEG Data}

SEEG signals were collected from the 5 patients with drug-resistant TLE (Table 1). These patients underwent stereotactic surgery for the intracranial implantation of depth electrodes (ADtech 8-16 contacts; multicontacts, interval 3-10 $\mathrm{mm}$; diameter $0.86 \mathrm{~mm}$ ).

SEEG signals were sampled at $512 \mathrm{~Hz}$ and recorded on a Nicolet system (Natus Medical Incorporated) with 8-10 contacts per intracranial electrode and a maximum of 100 recording channels. Data were digitized at a 16-bit resolution and stored on a hard drive. Simultaneous video and SEEG signals were recorded over a period of 7-14 days with the aim of validating the electroclinical features of seizures.

\section{Determining the Position and Depth of Electrodes and the Reconstruction of Cortical Surfaces}

Positions of implanted depth electrodes were determined postoperatively using high-resolution CT and structural MRI (without contrast). Specifically, within 24 hours of implantation, postoperative CT scans (Philips Machine Brilliance 64) were acquired using the following technical parameters: 64 slices; rotation duration of $1 \mathrm{sec}-$ ond with coverage of $16 \mathrm{~cm}$ per rotation; $60-\mathrm{kW}$ generator $(512 \times 512$ matrix), $120 \mathrm{kV}, 301 \mathrm{mAs}$; and axial slice thickness of $1 \mathrm{~mm}$. MRI data (a 1.5-T GE Signa HDxt system) were collected using an 8-channel phased-array head coil. This was achieved by obtaining a high-resolution, 3D, T1-weighted structural MR image taken along the axial plane using a fast spoiled gradient-recalled echo sequence (TR/TE/TI 10.02/4.28/0 msec, flip angle $15^{\circ}$, matrix 256 $\times 256$, bandwidth $31.2 \mathrm{kHz}$, field of view $256 \times 256 \mathrm{~mm}$, and axial slice thickness $1.0 \mathrm{~mm}$ ). FreeSurfer was used to reconstruct a 3D model of cortical surfaces for each patient based on data obtained from high-resolution structural MR images (obtained preoperatively). ${ }^{8}$ Postoperative CT scans were coregistered to corresponding preoperative MR images using the normalized mutual information- 
TABLE 1. Patient clinical and demographic information

\begin{tabular}{|c|c|c|c|c|c|c|c|c|}
\hline \multirow[b]{2}{*}{ Pt No. } & \multirow[b]{2}{*}{ Sex } & \multirow{2}{*}{$\begin{array}{c}\text { Age at Onset/Op } \\
(y r s)^{*}\end{array}$} & \multicolumn{2}{|r|}{ Sz† } & \multirow{2}{*}{$\begin{array}{c}\text { Electrodes } \\
\text { Type }\end{array}$} & \multirow{2}{*}{$\begin{array}{c}\text { No. of } \\
\text { Contacts/Electrodes§ }\end{array}$} & \multirow{2}{*}{$\begin{array}{l}\text { Sz Type } \\
\text { (no.)ף }\end{array}$} & \multirow[b]{2}{*}{ Imaging Finding } \\
\hline & & & No. & Duration (secs) & & & & \\
\hline 1 & M & $32 / 48$ & 3 & $152,172,191$ & Depth & $100 / 10$ & CPS (3) & Mesial temporal tumor \\
\hline 2 & $\mathrm{~F}$ & $15 / 17$ & 5 & $107,107,99,114,81$ & Depth & $80 / 8$ & CPS (4) + 2nd (1) & MRI negative \\
\hline 3 & M & $20 / 24$ & 4 & $111,112,120,115$ & Depth & $70 / 7$ & CPS (4) & MRI negative \\
\hline 4 & M & $5 / 16$ & 5 & $92,173,111,137,123$ & Depth & $60 / 7$ & CPS (5) & MRI negative \\
\hline 5 & $M$ & $24 / 41$ & 3 & $90,135,124$ & Depth & $90 / 10$ & CPS (3) & MRI negative \\
\hline
\end{tabular}

$\mathrm{Pt}=$ patient; $\mathrm{Sz}=$ seizure.

${ }^{*}$ Age at onset indicates the age at which seizures were first described by the patient.

$\dagger$ For each patient, the respective number of seizures were analyzed for each duration listed.

$\ddagger$ "Depth" refers to patients who received only orthogonal placement of intraparenchymal arrays of electrodes.

$\S$ The total number of intracranial electrodes and the number of electrodes involved at seizure onset are listed for the dominant seizure type.

I All seizure types were complex partial seizures (CPSs), which had secondary generalization (CPS + 2nd) and CPSs without secondary generalization (CPS); the number of each type of seizure for each patient is indicated.

based registration algorithm provided in ANALYZE 12.0 imaging software (Biomedical Imaging Resource, Mayo Foundation). ANALYZE 12.0 was also used to determine the locations of contacts from postoperative CT scans and to embed the contacts' locations within the reconstructed 3D model of the cortex.

\section{Construction of HFO Networks}

We constructed HFO networks from SEEG signals as follows. ${ }^{8}$ We began by pruning the SEEG segments associated with each seizure (retaining data from $60 \mathrm{sec}-$ onds prior to seizure onset to 30 seconds after seizure termination). We then performed a bipolar montage by subtracting monopolar SEEG signals recorded from two adjacent contacts. We then bandpass filtered the bipolar SEEG signals (third-order Butterworth, zero-phase digital filtering) in the HFO band of the $80-$ to $250-\mathrm{Hz}$ wavelength and notch-filtered (third-order Butterworth, zerophase digital filtering) the SEEG signals at 120, 180, and $240 \mathrm{~Hz}$. We then grouped the SEEG signals into 1-second windows with 0.5 seconds of overlap. Data from all electrodes within the same window were normalized to have a mean of zero and unit variance. Next, we calculated cross-correlation values for preprocessed SEEG signals between the two electrodes and selected the maximum correlation within time delays of \pm 0.5 seconds. We then determined the statistical significance of the maximum correlation value by performing an analytical procedure that interprets our choice of an extremum. ${ }^{19}$ The analytical procedure used the test statistic of the maximal crosscorrelation between a pair of time series to test the null hypothesis that two time series are uncorrelated against the alternative that they are correlated. It concentrated on computing an appropriate $\mathrm{p}$ value for the edge by the analytical procedure. It contained a comparison of a test statistic to a normal distribution. It scaled the test statistic by an estimated variance and then compared this scaled quantity to the standard normal distribution to calculate a $\mathrm{p}$ value. Then, we performed multiple significance tests using a linear step-up procedure to control the false detection rate with $q=0.05$. Finally, interelectrode cross-coefficients were measured between all pairs of electrodes for each 1-second window.

\section{Topological Analysis}

To analyze HFO networks derived from SEEG signals, we applied two network metrics: one that measures network density and another that considers component size. ${ }^{18,25,30}$ Network density is defined as the number of edges in the network divided by the total possible number of edges. A component comprises a subset of nodes from the network, in which each node is reachable from every other node. In this study, we were specifically interested in the largest components in the networks. In addition to network metrics, we computed the HFO energy associated with SEEG signals for each subject and seizure within each interval. To define the signal energy, we constructed time-frequency spectrograms from the magnitude of the coefficients of the complex Morlet wavelet transform. Then, we normalized the wavelet coefficient magnitudes with respect to frequency range of $80-250 \mathrm{~Hz}$.

$$
\mathrm{W}_{\text {normalized }}(f, t)=\left(\left|\mathrm{W}_{\text {norm }}(f, t)\right|-\mu(f)_{\text {baseline }}\right) / \sigma(f)_{\text {baseline }}
$$

The variables $\mu$ and $\sigma$ denote the mean and standard deviation of the SEEG baseline segment of magnitudes for each corresponding frequency $f$. Values for each frequency were calculated from 5 SEEG segments (each of which was 1 second in length) of baseline SEEG activity that had been recorded in the preictal period (at least 30 seconds before onset). The 5 SEEG segments of baseline SEEG activity were selected to guarantee each segment that minimized the effect of transient events and to obtain representative mean and standard deviation values for electrical activities at various frequencies. Normalized time-frequency distributions of wavelets for each electrode were averaged across the 1-second windows and also across HFO bands of the $80-250 \mathrm{~Hz}$ to relate average SEEG energy. The average SEEG energy for each subject was summed across the 1-second windows and across all channels to relate overall energy.

\section{Statistical Analysis}

Seizure duration varied among seizures and subjects. Therefore, for each patient, we normalized seizure duration data by dividing each seizure into 10 intervals of equal length, labeled I1 to I10. We assumed that seizures 
evolved in a typical manner and that the duration of seizure evolution was likely to vary from subject to subject or even from seizure to seizure. ${ }^{20}$ In addition to the 10 ictal intervals, we also analyzed network properties during preictal intervals (from 30 seconds before seizure onset to seizure onset, labeled as -1 ) and postictal intervals (from seizure termination to 30 seconds after seizure termination, labeled as +1). We applied one-way ANOVA to determine significant changes in the network measures and signal energy across the intervals for the entire seizures. A multiple comparison procedure was also performed to identify the pairs of intervals that exhibited significant differences. Differences were considered to be statistically significant at a level of $\mathrm{p}=0.05$.

\section{Results}

To validate the electroclinical features of seizures, video and SEEG signals were recorded simultaneously for an average period of 9 days (range 7-14 days). Data from a total of 20 seizures were collected from 5 patients who presented with drug-resistant TLE (Table 1). All seizures were complex partial seizures $(n=20)$, except one seizure showed secondary generalization $(\mathrm{n}=1)$. The median duration of seizures was 115 seconds (range 81-191 seconds) (Table 1). After dividing seizures into 10 intervals of equal length (labeled I1-I10), normalized interval duration ranged from 8.1 to 19.1 seconds.

After stereotactically implanting depth electrodes, the 5 patients had $100,80,70,70$, and 90 contacts, respectively (8-16 contacts per electrode; multiple contacts at intervals of 3-10 mm), and each of these contacts was individually analyzed. For all 5 patients, we reconstructed 3D maps that included the positions of depth electrodes. Specifically, electrodes were mapped in individual brains extracted from the high-resolution structural images of each patient. Figure 1A illustrates electrode placement in one patient; the hippocampus in the figure is labeled to facilitate better visualization. We observed that the HFO network constantly changed throughout seizure evolution (Fig. 1B-D and Video 1).

VIDEO 1. An illustrated case to show the ictal HFO networks of TLE. Copyright Taipei Veterans General Hospital. Published with permission. Click here to view.

Figure 2 illustrates these changes dynamically, by showing network components at various intervals (I1-I10) of the same seizure as Fig. 1. As shown in Fig. 2, components in the mesial temporal structure (e.g., the hippocampus and amygdala) grew slowly during intervals I1I3. However, HFO network components accumulated a large number of edges during intervals I4-I7. At the end of seizure (I8-I10), the number of edges in HFO network components had begun to decrease, and components had begun to separate into several smaller ones. Figure 3A shows the changes in network density averaged across all 20 seizures. As shown in the figure, at seizure onset, HFO networks were characterized by a growing number of edges. This number peaked between intervals I6-I8 and then gradually decreased as the seizure ended.

Figure 2 also illustrates changes in HFO network components for a single seizure. Prior to seizure onset $(-1)$, there were multiple components, which indicated a scattered HFO network. During the early seizure period (I1I3), several nodes in mesial temporal structures were found to connect together to form a small component. During the middle seizure period, the majority of nodes gradually joined the large component, which encompassed parts of the mesial, lateral, and extratemporal regions of the brain. Following ictal termination, however, the largest component shrank, thereby fracturing the network. The graphs in Fig. $3 \mathrm{C}$ and $\mathrm{D}$ show common changes in component numbers, as well as the percentage of nodes that belonged to the largest component. Note that the number of components gradually decreased following seizure onset, reached a minimum during intervals I7-I8, and then gradually increased until the end of the seizure. Conversely, almost $80 \%$ of nodes joined the largest component during intervals I7I8; however, large components fractured into smaller ones at the end of seizures. In summary, as the graphs in Fig. 3C and 3D clearly demonstrate, HFO network components randomly appeared prior to seizure onset, merged during the seizure, and then fractured after the seizure had ended.

Statistical analysis revealed a significant increase in network density during intervals I5-I10 ( $<0.05$ at I5-I10) (Fig. 3A). Similarly, a significant increase in overall energy release was also noted during intervals I3-I8, compared to the preictal period (Fig. 3B). The onset of significant increase of energy release (interval I3) was earlier than that of network density (interval I5), and the peak of energy release (interval I6) was also earlier than that of network density (interval I8). Nonetheless, network density and energy release appear to follow the same general pattern, and both network density and energy release were found to decrease at the end of the seizure. In subnetwork analysis, the number of components was found to continuously decrease following seizure onset, and this decrease reached statistical significance during intervals I3-I10 (Fig. 3C). During seizure evolution, most of the nodes became joined to a single dominant component, and the percentage of nodes that belonged to the largest component grew significantly (Fig. 3D). During intervals I2-I10, as well as after seizure termination, nearly half of all nodes were connected to the largest component, thereby dominating the network.

\section{Surgical Outcome After Resection Surgery}

Table 2 shows the surgical outcome at the 2-year follow-up. These 5 patients underwent SEEG to identify their seizure onset zone, irritative zone, and possible EZ. Four of them were MRI-negative cases. After localizing the HFO involving and evolving region in the initial seizure onset (I1-I3), the resection surgeries were arranged. Four of the patients underwent selective amygdalohippocampectomy, and one underwent anterior temporal lobe lobectomy according to their SEEG finding. Except for one ganglioglioma, the other pathologies showed neuronal loss and gliosis, which are common histological features in patients with mesial temporal sclerosis. In the patient who underwent anterior temporal lobe lobectomy, the lateral cortex showed gliosis. These 5 patients had regular follow-up for at least 2 years. At the time point of 2 years after resection, all 5 patients were seizure free and no anticonvulsant medications were being taken. 
Peng et al.
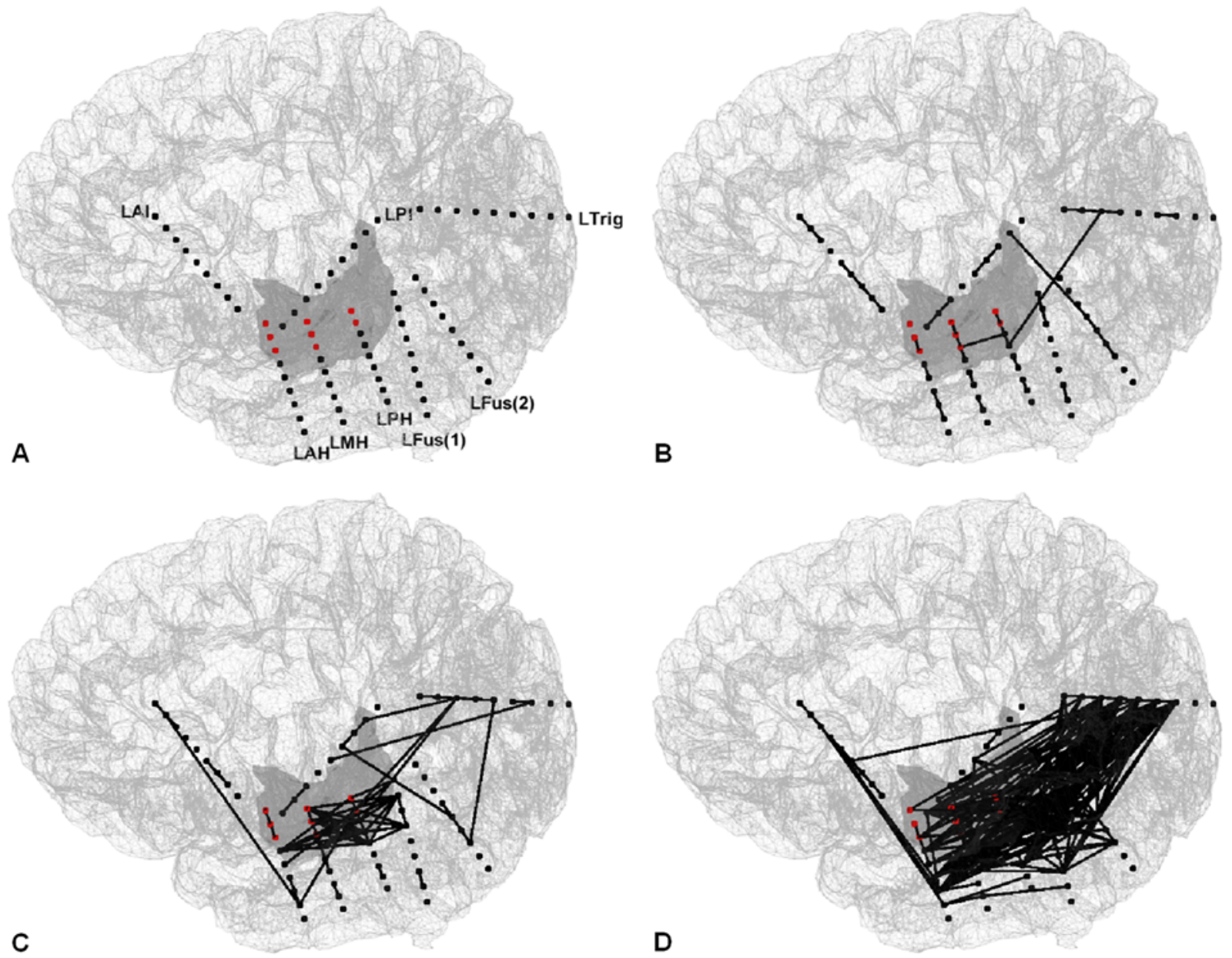

FIG. 1. Image showing individual nodes in a reconstructed cortical surface (A). Red nodes are located in the left hippocampus. Representative networks from a single seizure (B) prior to seizure onset (-1), at seizure onset (interval I1) (C), and in the middle of seizure (interval I6) (D). LAH = left anterior hippocampus; LAI = left anterior insula; LFus(1) = left fusiform gyrus (1); LFus(2) = left fusiform gyrus (2); LMH = left middle hippocampus; LPH = left posterior hippocampus; $L P I=$ left posterior insula; $L$ Trig = left trigone. Figure is available in color online only.

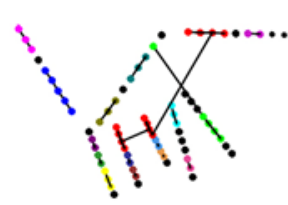

$-1$

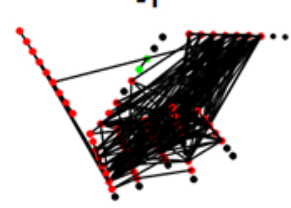

16

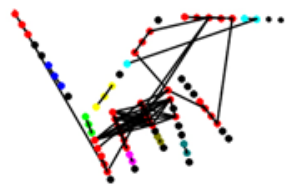

11

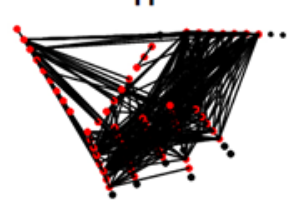

17

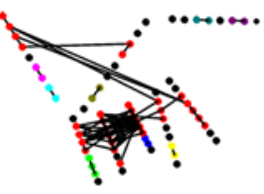

12

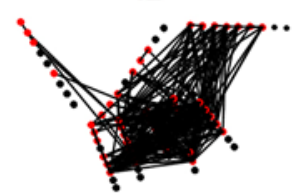

18

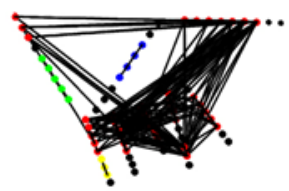

13

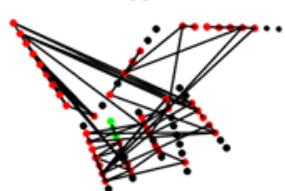

19

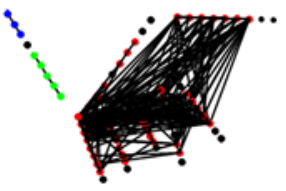

14

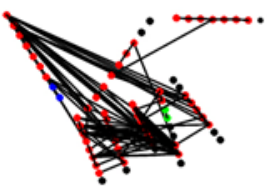

110

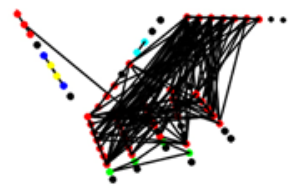

15

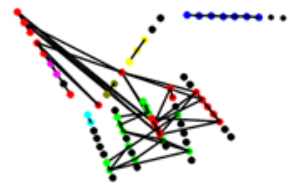

$+1$

FIG. 2. Examples of an HFO network and connected components during preictal (-1), ictal (I1-I10), and postictal (+1) intervals. Data shown in this figure are from a single subject and seizure. Circles denote nodes and black lines denote edges. Electrodes have been colored to signify their associations with components, such that all electrodes of the same color belong to the same component; red denotes the largest component, and gray denotes isolated electrodes. During the middle of a seizure, most of the nodes gradually become connected with the largest component, which, in this image, covers portions of the mesial, extratemporal, and lateral temporal lobe. Figure is available in color online only. 

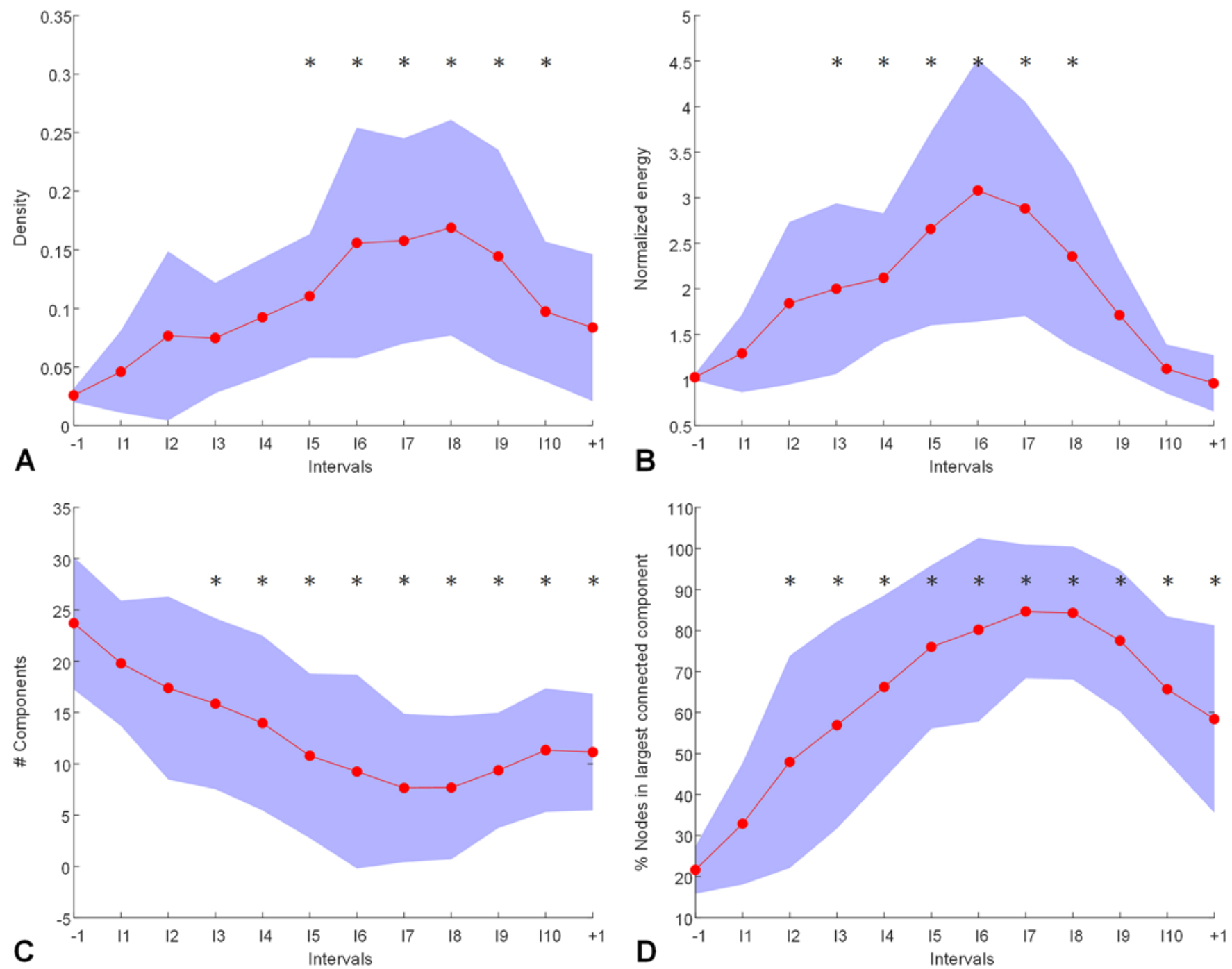

FIG. 3. A: Network density averaged across all subjects and seizures and adjusted for differences in subjects. Network density is shown at 12 time intervals, including 1 preictal interval $(-1), 10$ ictal intervals (I1-|10), and 1 postictal interval $(+1)$. Statistically significant increases in network density (compared with preictal values) were recorded at I5-I10. B: Plots of normalized signal energy for each interval averaged across all subjects and seizures. Similar to network density, signal energy during $13-18$ showed a statistically significant increase compared with preictal values. C and D: Conversely, the average number of connected components significantly decreased during intervals $|3-| 10$ and at $+1(C)$, while the average percentage of nodes in the largest connected component significantly increased during I2-I10 and at +1 (D). For each interval, the red circles and blue regions indicate the mean and standard deviation, respectively. Asterisks indicate that values are significantly different from those recorded during the preictal state. Figure is available in color online only.

\section{Discussion}

\section{Use of SEEG to Explore Dynamic Topologies in HFO Networks}

The use of SEEG to identify the EZ in patients with drug-resistant epilepsy has become increasingly popular in the last decade. The exploration of dynamic topologies makes it possible for SEEG to delineate the hypothetical epileptic network in 3 dimensions using multiple intracerebral electrodes. Therefore, cortical structures that have been implicated in the generation and early propagation of seizures can be mapped with precise stereotactic guidance, and dynamic topologies of cortical and subcortical functional networks can be established.
By extracting and quantifying spatiotemporal data from complicated signals recorded directly within the epileptic brain, SEEG analysis can help to define and elucidate ictogenesis. ${ }^{2,10}$ In this study, the duration and frequency of SEEG signals were analyzed to identify changes occurring during the transition from the interictal to the ictal state. Specifically, SEEG is able to identify changes in the relationship between the EZ, the ictal onset zone, the irritative zone, and the symptomatic zone., ${ }^{1,3,11,32}$ Previous research into nonlinear correlations or coherence demonstrated that preferential interactions occur between subregions of the mesial temporal lobe during the generation of epileptic patterns. Between these subregions, HFOs comprise the 
TABLE 2. Seizure outcome after resection

\begin{tabular}{|c|c|c|c|c|c|c|c|}
\hline $\begin{array}{l}\mathrm{Pt} \\
\text { No. }\end{array}$ & Sex & $\begin{array}{l}\text { Imaging } \\
\text { Finding }\end{array}$ & $\begin{array}{l}\text { Origin of SEEG } \\
\text { Changes }\end{array}$ & $\begin{array}{l}\text { Resection } \\
\text { Extent }\end{array}$ & $\begin{array}{l}\text { Resection Extent Correlated to } \\
\text { Ictal Network From SEEG }\end{array}$ & Pathology & $\begin{array}{c}\text { Surgical Outcome } \\
(2-y r \text { FU) }\end{array}$ \\
\hline 1 & M & $\begin{array}{l}\text { Mesial temporal } \\
\text { tumor }\end{array}$ & Lt mesial temporal & Lt SAH & $\begin{array}{l}\text { Initial HFO involving area on } \\
\text { ictal onset (I1-2) }\end{array}$ & Ganglioglioma & Engel class I \\
\hline 2 & $\mathrm{~F}$ & MRI negative & Lt mesial temporal & Lt SAH & $\begin{array}{l}\text { Initial HFO involving area on } \\
\text { ictal onset (I1-3) }\end{array}$ & $\begin{array}{l}\text { Neuronal loss \& gliosis (me- } \\
\text { sial temporal sclerosis) }\end{array}$ & Engel class I \\
\hline 3 & M & MRI negative & Rt mesial temporal & Rt SAH & $\begin{array}{l}\text { Initial HFO involving area on } \\
\text { ictal onset (I1-3) }\end{array}$ & $\begin{array}{l}\text { Neuronal loss \& gliosis (me- } \\
\text { sial temporal sclerosis) }\end{array}$ & Engel class I \\
\hline 4 & M & MRI negative & $\begin{array}{l}\text { Lt mesial temporal ( } 1 \\
\text { event) \& lat temporal } \\
\text { cortex ( } 4 \text { events) }\end{array}$ & Lt ATL & $\begin{array}{l}\text { Initial HFO involving area on } \\
\text { ictal onset (I1-3); both mesial } \\
\text { temporal structure \& lat cortex }\end{array}$ & $\begin{array}{l}\text { Neuronal loss \& gliosis (me- } \\
\text { sial temporal) \& gliosis } \\
\text { (lateral cortex) }\end{array}$ & Engel class I \\
\hline 5 & M & MRI negative & Lt mesial temporal & Lt SAH & $\begin{array}{l}\text { Initial HFO involving area on } \\
\text { ictal onset (I1-2) }\end{array}$ & $\begin{array}{l}\text { Neuronal loss \& gliosis (me- } \\
\text { sial temporal sclerosis) }\end{array}$ & Engel class I \\
\hline
\end{tabular}

$\mathrm{ATL}=$ anterior temporal lobectomy; FU = follow-up; SAH = selective amygdalohippocampectomy.

most readily distinguishable biomarker. Indeed, early electrodecremental events that involve high-frequency activity (i.e., HFOs that occur between 80 and $250 \mathrm{~Hz}$ ) have been associated with the EZ. ${ }^{26,27,36}$ Furthermore, even higherfrequency oscillations, known as fast HFOs $(250-500 \mathrm{~Hz})$, have been observed in the EZ of patients with TLE during ictal and interictal periods. ${ }^{5,12}$ Rapid changes in frequency have been shown to comprise highly specific and sensitive spectrographic signatures of epileptic seizure activity and can therefore be used as markers of EEG seizure. ${ }^{22,28}$

In this study, we sought to improve access to the epileptogenic network using 3D electrodes implanted based on the SEEG methodology. We then investigated the dynamic topologies of cortical and subcortical functional networks in order to identify topology changes that consistently occur during seizure propagation.

\section{Consistent Topology Changes Imply a Common Ictogenetic Pattern in TLE}

In this study, we enrolled 5 patients who suffered from a total of 20 seizures. Although these seizures resulted from multiple etiologies, we observed that the evolution of HFO network structures followed a consistent pattern over the course of seizures. The network density values across the ictal interval (I1-I10) of individual seizures showed that most fall within 2 standard deviations of the corresponding mean network density. There was consistent pattern in the graph from seizure to seizure. Specifically, at the onset of the seizure, HFOs were not intense throughout the entire SEEG network. Rather, HFOs were recorded only by scattered electrodes, and subnetworks were not synchronous. These observations suggest that high-frequency activities are not uniformly hypersynchronous at the beginning of a seizure. Instead, synchrony is strongest during the middle of a seizure and gradually decreases toward seizure termination (Fig. 3A). These results indicated that the increase of synchronization with high-amplitude and low-frequency oscillations is characteristic of seizure progression, which is consistent with previous studies., ${ }^{920}$ Our result is also consistent with the previous two reports about the ictogenesis in TLE of a rat model. Both demonstrated the patterns of incrementally increasing fast ripple power are consistent with observations in rats with experimental hippocampal epilepsy. They also suggested that limbic seizures arise when small clusters of synchronously bursting neurons increase in size, coalesce, and reach a critical mass for propagation. . $^{21,31}$

Greater synchronization in the HFO network is correlated with higher energy release, which also provides a crude characterization of network topology (Fig. 3B). To explore the structure of the HFO network in detail, we used graph theory and associated analytical techniques to elucidate a consistent pattern of HFO network evolution, from seizure onset to seizure termination. At the seizure onset, data from depth electrodes revealed that there were several components within the network (Figs. 2 and 3C). Over the course of the seizure, a large subnetwork of connected nodes emerged (i.e., the dominant component). As the dominant component continued to merge with smaller components, the overall number of components decreased. As the seizure neared termination, the dominant component fractured, and the distribution pattern of components returned to its interictal state (Fig. 3C and D).

\section{Implications for Epilepsy Surgeries}

Current standard practice in epilepsy surgery involves locating the focal EZ and attempting to resect it. Resection provides a favorable outcome for patients with focal drug-resistant epilepsy; however, about half of the patients with drug-resistant epilepsy are not suitable candidates for resection as determined in presurgical evaluation. Nonetheless, new approaches to epilepsy treatment are rapidly developing, including cortical stimulation in response to seizure activity ${ }^{24}$ and neuromodulation of specific brain regions that have been identified as "hubs" of seizure activity. Either way, the findings in our study should be beneficial to researchers and physicians working to improve various epileptic treatment modalities, including resection, cortical stimulation, or neuromodulation-based treatments that are responsive to network topology.

The HFO network is undergoing constant change; therefore, treatment strategies must target the HFO network at the appropriate time. The most efficient way to treat epilepsy patients may be to prevent the formation of the largest 
component in the middle of a seizure (i.e., to prevent the increase in network density that characterizes the middle of the ictal period). An alternative approach would involve blocking specific brain regions that have been identified as "hubs" of the HFO network. It is conceivable that appropriate electrical stimulation could block electrical progression through the network, thereby terminating the seizure prior to clinical manifestations.

\section{Brain Plasticity and Epileptogenicity}

HFOs are good biomarkers for the EZ in focal epilepsy. Resecting the areas of the brain where HFOs have been recorded often leads to good postsurgical outcomes. This suggests that HFOs may play a role in epileptogenesis and ictogenesis. ${ }^{35}$ In the current study, we observed the presence of HFOs in temporal lobe seizures, where they initially localized in mesial temporal structures and then propagated to the lateral and extratemporal regions. We hypothesized that these HFO patterns, which were present over recurrent seizures, could play a role in epileptogenesis by causing kindling and rewiring the brain. ${ }^{23}$ Our findings help to elucidate the epileptogenic network that characterizes TLE.

\section{Study Limitations}

As with any invasive procedure, there are inherent limitations to SEEG methodology. First, because the limited number of contacts were located in the brain, which might not cover the entire epileptic network, sampling error could have occurred when the network was scaled up. Second, we attempted to condense complicated network features to several scalar values (e.g., network density and number of components) in order to explain and compare network attributes across the patient population. Although our results appear to be meaningful, the simplified tools we used may have left out details of focal network features of individual seizures, which a more comprehensive analysis could potentially have captured. Concentrating on these focal characteristics may disregard the overall features of bihemispheric seizure activity that emerge during seizure propagation.

\section{Conclusions}

We identified common changes that occur in the epileptic brain over the course of a seizure. Specifically, these changes pertain to the following: 1) the HFO network, 2) the amount of energy released, 3) the number of components, and 4) the percentage of nodes that are found in the largest connected component. The nodular disorganization of the HFO network precedes the onset of a seizure, becomes organized in the middle of the seizure, becomes fractured at the termination of the seizure, and returns to baseline distribution in the postictal period. Nodular disorganization is based on cortical HFOs, which are generated from synaptic and nonsynaptic mechanisms. ${ }^{17}$ These are located between principal cortical neurons and are independent of the mechanisms that drive observable electrographic seizure activity, such as spikes and sharp waves. In this sense, spatiotemporal maps of HFO networks at the onset of a seizure can be considered biomarkers of ictogenesis driving a network of cortical neurons to ultimately generate epileptic seizure activity. ${ }^{4,16}$

\section{Acknowledgments}

This work was financially supported in part by the Ministry of Science and Technology, Taiwan, under the project MOST 1062221-E-009-068 and in part by the Center for Neuromodulation Medical Electronics Systems from The Featured Areas Research Center Program within the framework of the Higher Education Sprout Project by the Ministry of Education in Taiwan. The work was also supported from Veterans General Hospitals and University System of Taiwan Joint Research Program (VGHUST107G1-4-1).

\section{References}

1. Bartolomei F, Chauvel P, Wendling F: Epileptogenicity of brain structures in human temporal lobe epilepsy: a quantified study from intracerebral EEG. Brain 131:1818-1830, 2008

2. Bartolomei F, Lagarde S, Wendling F, McGonigal A, Jirsa V, Guye M, et al: Defining epileptogenic networks: Contribution of SEEG and signal analysis. Epilepsia 58:1131-1147, 2017

3. Bartolomei F, Wendling F, Bellanger JJ, Régis J, Chauvel P: Neural networks involving the medial temporal structures in temporal lobe epilepsy. Clin Neurophysiol 112:1746-1760, 2001

4. Bikson M, Fox JE, Jefferys JG: Neuronal aggregate formation underlies spatiotemporal dynamics of nonsynaptic seizure initiation. J Neurophysiol 89:2330-2333, 2003

5. Bragin A, Engel J Jr, Wilson CL, Fried I, Buzsáki G: Highfrequency oscillations in human brain. Hippocampus 9:137142,1999

6. Cossu M, Cardinale F, Castana L, Citterio A, Francione S, Tassi L, et al: Stereoelectroencephalography in the presurgical evaluation of focal epilepsy: a retrospective analysis of 215 procedures. Neurosurgery 57:706-718, 2005

7. Cotic M, Zalay OC, Chinvarun Y, del Campo M, Carlen PL, Bardakjian BL: Mapping the coherence of ictal high frequency oscillations in human extratemporal lobe epilepsy. Epilepsia 56:393-402, 2015

8. Dale AM, Fischl B, Sereno MI: Cortical surface-based analysis. I. Segmentation and surface reconstruction. Neuroimage 9:179-194, 1999

9. Gnatkovsky V, Francione S, Cardinale F, Mai R, Tassi L, Lo Russo G, et al: Identification of reproducible ictal patterns based on quantified frequency analysis of intracranial EEG signals. Epilepsia 52:477-488, 2011

10. Gonzalez-Martinez JA: The stereo-electroencephalography: the epileptogenic zone. J Clin Neurophysiol 33:522-529, 2016

11. Gotman J, Levtova V: Amygdala-hippocampus relationships in temporal lobe seizures: a phase-coherence study. Epilepsy Res 25:51-57, 1996

12. Jacobs J, LeVan P, Chander R, Hall J, Dubeau F, Gotman $\mathrm{J}$ : Interictal high-frequency oscillations $(80-500 \mathrm{~Hz})$ are an indicator of seizure onset areas independent of spikes in the human epileptic brain. Epilepsia 49:1893-1907, 2008

13. Jacobs J, Zijlmans M, Zelmann R, Chatillon CE, Hall J, Olivier A, et al: High-frequency electroencephalographic oscillations correlate with outcome of epilepsy surgery. Ann Neurol 67:209-220, 2010

14. Jirsch JD, Urrestarazu E, LeVan P, Olivier A, Dubeau F, Gotman J: High-frequency oscillations during human focal seizures. Brain 129:1593-1608, 2006

15. Jiruska P, Alvarado-Rojas C, Schevon CA, Staba R, Stacey $\mathrm{W}$, Wendling F, et al: Update on the mechanisms and roles of high-frequency oscillations in seizures and epileptic disorders. Epilepsia 58:1330-1339, 2017

16. Jiruska P, Csicsvari J, Powell AD, Fox JE, Chang WC, Vreugdenhil M, et al: High-frequency network activity, global increase in neuronal activity, and synchrony expansion precede epileptic seizures in vitro. J Neurosci 30:5690-5701, 2010 
17. Jiruska P, Powell AD, Chang WC, Jefferys JG: Electrographic high-frequency activity and epilepsy. Epilepsy Res 89:60-65, 2010

18. Kolaczyk ED: Statistical Analysis of Network Data: Methods and Models. New York: Springer, 2009

19. Kramer MA, Eden UT, Cash SS, Kolaczyk ED: Network inference with confidence from multivariate time series. Phys Rev E Stat Nonlin Soft Matter Phys 79:061916, 2009

20. Kramer MA, Eden UT, Kolaczyk ED, Zepeda R, Eskandar EN, Cash SS: Coalescence and fragmentation of cortical networks during focal seizures. J Neurosci 30:10076-10085, 2010

21. Lévesque M, Salami P, Gotman J, Avoli M: Two seizureonset types reveal specific patterns of high-frequency oscillations in a model of temporal lobe epilepsy. J Neurosci 32:13264-13272, 2012

22. Molaee-Ardekani B, Benquet P, Bartolomei F, Wendling F: Computational modeling of high-frequency oscillations at the onset of neocortical partial seizures: from 'altered structure' to 'dysfunction'. Neuroimage 52:1109-1122, 2010

23. Morimoto K, Fahnestock M, Racine RJ: Kindling and status epilepticus models of epilepsy: rewiring the brain. Prog Neurobiol 73:1-60, 2004

24. Morrell M: Brain stimulation for epilepsy: can scheduled or responsive neurostimulation stop seizures? Curr Opin Neurol 19:164-168, 2006

25. Newman M: The structure and function of complex networks. SIAM Rev 45:167-256, 2003

26. Pail M, Řehulka P, Cimbálník J, Doležalová I, Chrastina J, Brázdil M: Frequency-independent characteristics of highfrequency oscillations in epileptic and non-epileptic regions. Clin Neurophysiol 128:106-114, 2017

27. Perucca P, Dubeau F, Gotman J: Intracranial electroencephalographic seizure-onset patterns: effect of underlying pathology. Brain 137:183-196, 2014

28. Schiff SJ, Colella D, Jacyna GM, Hughes E, Creekmore JW, Marshall A, et al: Brain chirps: spectrographic signatures of epileptic seizures. Clin Neurophysiol 111:953-958, 2000

29. Talairach J, Bancaud J, Szikla G, Bonis A, Geier S, Vedrenne $\mathrm{C}$ : [New approach to the neurosurgery of epilepsy. Stereotaxic methodology and therapeutic results. 1. Introduction and history.] Neurochirurgie 20 (Suppl 1):1-240, 1974 (Fr)

30. Wasserman S, Faust K: Social Network Analysis: Methods and Applications. Cambridge, UK: Cambridge University Press, 1994

31. Weiss SA, Alvarado-Rojas C, Bragin A, Behnke E, Fields T, Fried I, et al: Ictal onset patterns of local field potentials, high frequency oscillations, and unit activity in human mesial temporal lobe epilepsy. Epilepsia 57:111-121, 2016
32. Wendling F, Bartolomei F, Bellanger JJ, Bourien J, Chauvel P: Epileptic fast intracerebral EEG activity: evidence for spatial decorrelation at seizure onset. Brain 126:1449-1459, 2003

33. Worrell GA, Parish L, Cranstoun SD, Jonas R, Baltuch G, Litt B: High-frequency oscillations and seizure generation in neocortical epilepsy. Brain 127:1496-1506, 2004

34. Wu JY, Sankar R, Lerner JT, Matsumoto JH, Vinters HV, Mathern GW: Removing interictal fast ripples on electrocorticography linked with seizure freedom in children. Neurology 75:1686-1694, 2010

35. Zijlmans M, Jiruska P, Zelmann R, Leijten FS, Jefferys JG, Gotman J: High-frequency oscillations as a new biomarker in epilepsy. Ann Neurol 71:169-178, 2012

36. Zweiphenning WJ, van 't Klooster MA, van Diessen E, van Klink NE, Huiskamp GJ, Gebbink TA, et al: High frequency oscillations and high frequency functional network characteristics in the intraoperative electrocorticogram in epilepsy. Neuroimage Clin 12:928-939, 2016

\section{Disclosures}

The authors report no conflict of interest concerning the materials or methods used in this study or the findings specified in this paper.

\section{Author Contributions}

Conception and design: Lee, Peng, Chou, Yu. Acquisition of data: Lee, Chou, Yu, C Chen, Yen, Kwan, Hsu, Lin, HH Chen. Analysis and interpretation of data: Peng. Drafting the article: Lee, Peng. Reviewed submitted version of manuscript: Lee, Peng, Chou. Statistical analysis: Peng. Administrative/technical/material support: Lee. Study supervision: Lee, Peng, Chou.

\section{Supplemental Information \\ Videos \\ Video 1. https://vimeo.com/284133130.}

\section{Correspondence}

Cheng-Chia Lee: Neurological Institute, Taipei Veterans General Hospital, Taipei, Taiwan. yfnaughty@gmail.com. 